\title{
Optimization of busulfan dosing regimen in pediatric patients using population pharmacokinetic model incorporating GST polymorphisms
}

\author{
Jin-jie Yuan ${ }^{1}$, Ning Sun ${ }^{1}$, Xin-ying Feng ${ }^{1}$, Huan $\mathrm{He}^{2}$, Guang-hua Zhu ${ }^{1}$, and Li-Bo Zhao ${ }^{3}$ \\ ${ }^{1}$ Affiliation not available \\ ${ }^{2}$ Beijing Children's Hospital, Capital Medical University, National Center for Children's \\ Health, Beijing 10045, China \\ ${ }^{3}$ Peking University
}

September 17, 2020

\begin{abstract}
Aim: The aim of this study was to develop a population pharmacokinetic (PPK) model in Chinese children for intravenous busulfan, and to develop a novel busulfan dosing regimen to support better area under the concentration-time curve (AUC) targeting. Methods: We collected busulfan concentration-time samples from 69 children who received intravenous busulfan prior to allogeneic hematopoietic stem cell transplantation (allo-HSCT). A population pharmacokinetic model for busulfan was developed by nonlinear mixed effect modelling and was validated by an external dataset $(\mathrm{n}=14)$. A novel busulfan dosing regimen was developed through simulation on 1000 patients. Limited Sampling Strategy (LSS) was established by the Bayesian forecasting. Absolute Prediction Error (APE), Mean Absolute Prediction Error (MAPE) and relative Root Mean Squared Error (rRMSE) were calculated to evaluate predictive accuracy. Results: A one-compartment model with first-order elimination best described the data. GSTA1 genotypes, BSA and AST were found to be significant covariates of Bu clearance, and BSA had remarkable impact of the volume. Moreover, recommended dose regimens for children with different GSTA1 genotypes and BSA were developed with a perfect AUC targeting. A two-point LSS, two hours and four hours after dosing, behaved well with acceptable prediction precision. Conclusion: This study developed a PPK model for busulfan that firstly incorporated GSTA1 genotypes in an Asian pediatric population. We recommend a BSA-based dosing for personalizing busulfan therapy in pediatric population. Additionally, an optimal LSS (C2h and $\mathrm{C} 4 \mathrm{~h}$ ) provides convenience for therapeutic drug monitoring (TDM) in the future.
\end{abstract}

\begin{abstract}
Aim : The aim of this study was to develop a population pharmacokinetic (PPK) model in Chinese children for intravenous busulfan, and to develop a novel busulfan dosing regimen to support better area under the concentration-time curve (AUC) targeting.

Methods : We collected busulfan concentration-time samples from 69 children who received intravenous busulfan prior to allogeneic hematopoietic stem cell transplantation (allo-HSCT). A population pharmacokinetic model for busulfan was developed by nonlinear mixed effect modelling and was validated by an external dataset $(n=14)$. A novel busulfan dosing regimen was developed through simulation on 1000 patients. Limited Sampling Strategy (LSS) was established by the Bayesian forecasting. Absolute Prediction Error (APE), Mean Absolute Prediction Error (MAPE) and relative Root Mean Squared Error (rRMSE) were calculated to evaluate predictive accuracy.
\end{abstract}

Results : A one-compartment model with first-order elimination best described the data. GSTA1 genotypes, 
BSA and AST were found to be significant covariates of Bu clearance, and BSA had remarkable impact of the volume. Moreover, recommended dose regimens for children with different GSTA1 genotypes and BSA were developed with a perfect AUC targeting. A two-point LSS, two hours and four hours after dosing, behaved well with acceptable prediction precision.

Conclusion : This study developed a PPK model for busulfan that firstly incorporated GSTA1 genotypes in Chinese pediatric population. We recommend a BSA-based dosing for personalizing busulfan therapy in pediatric population. Additionally, an optimal LSS $\left(\mathrm{C}_{2 \mathrm{~h}}\right.$ and $\left.\mathrm{C}_{4 \mathrm{~h}}\right)$ provides convenience for therapeutic drug monitoring (TDM) in the future.

KEYWORDS : busulfan, HSCT, individualized therapy, population pharmacokinetics, pediatric patients

\section{Introduction}

Busulfan $(\mathrm{Bu})$ is a common alkylating agent, which can bind to the guanine of intracellular DNA to damage its structure and function (1). Bu-based conditioning schemes are regarded as the cornerstone of allogeneic hematopoietic stem cell transplantation (allo-HSCT) because of their myeloablative activity $(1,2)$. However, IV $\mathrm{Bu}$ usually leads to large variability in pharmacokinetics and the treatment window is narrow. Higher exposure (expressed as area-under-the-curve of $\left.0-6 \mathrm{~h}, \mathrm{AUC}_{0-6 \mathrm{~h}}\right)(>1350 \mu \mathrm{M} \cdot \mathrm{min})$ or low $\mathrm{AUC}_{0-6 \mathrm{~h}}(<$ $900 \mu \mathrm{M} \cdot \mathrm{min})$ of $\mathrm{Bu}$ may lead to a higher probability of sinusoidal obstructive syndrome (SOS) or acute graft-versus-host disease (aGVHD) (3-5). The exposure and clinical outcomes of Bu are largely affected by different conditioning regimen agents, which has been confirmed in children receiving $\mathrm{Bu} /$ cyclophosphamide (Bu/CTX), Bu/fludarabine (Bu/FLU) and Bu/ cyclophosphamide/etoposide (Bu/CTX/VP16) (6-8). Thus, therapeutic drug monitoring (TDM) for busulfan is the integral component of HSCT. Personalized Bu dosing via TDM based on the first-dose pharmacokinetics (PK) could make contribution to lower the occurrence of toxicity and to improve rate of engraftment $(9,10)$.

$\mathrm{Bu}$ is metabolized by the formation of a glutathione conjugate in the liver $(11,12)$. This reaction is primarily catalyzed by glutathione S-transferase (GST) enzymes, such as GSTA1 ,GSTM1 , GSTT1 and GSTP1 (13).GSTA1 is the predominant GST enzyme involved in Bu metabolism, and the activity of GSTM1 is close to the half of GSTA1. However, the activity of GSTT1 and GSTP1 is relatively weak $(2,14)$. Hence, polymorphisms in the GSTA1 or GSTM1 isoenzymes would more likely affect Bu metabolism.

Studies about the relationships between GSTA1 polymorphisms and Bu PK have yielded inconsistent results. Although it has been reported that GSTA1 haplotypes were not significant influence factors of intravenous (IV) Bu clearance (15), Yin et al. (16) stated adult patients with the GSTA1 ${ }^{*} A /{ }^{*} B$ genotype had a significantly higher AUC, higher peak concentration $\left(\mathrm{C}_{\max }\right)$ and lower clearance $(\mathrm{CL})$. Pediatric patients have an increased Bu clearance when compared with adults (17), which is partially caused by higher expression or activity of GST enzymes in children $(18,19)$. Research on the relationship between GSTA1 polymorphism and PK in children is also controversial. Ansari et al. (20) reported that $G S T A 1{ }^{*} A{ }^{*} A$ was associated with lower drug concentration and more extensive metabolism. Conversely, Zwaveling et al. (21) asserted that GSTA1 polymorphisms had no influence on population PK parameters of IV $\mathrm{Bu}$ in children undergoing HSCT. In addition to age, the activity of GST enzymes is also affected by race. Minor allele frequencies (MAF) taken from HapMap were reported to be differing between Caucasian and Asian populations. Until now, the correlation between GST polymorphisms and PK in Chinese children has not been reported.

It has been suggested that, apart from GSTs polymorphism, the effect of age, body-weight (WT), primary disease, hepatic function, renal function and drug interactions (fludarabine and phenytoin) may partly explain inter-individual variability on Bu PK (22-25). Little is known about correlates of Bu PK in Chinese children, which may be potential factors to optimize dosage of $\mathrm{Bu}$.

The aim of this study was thus to build a population pharmacokinetic (PPK) model with data from pediatric patients, so as to present the PK feature of $\mathrm{Bu}$, to reveal the variability of PK parameters, and to identify the potential contribution of covariates on the disposition of Bu. Furthermore, Maximum A Posteriori (MAP) Bayesian forecasting made use of a PPK model and limited number of samples to forecast $\mathrm{AUC}_{0-6 \mathrm{~h}}$ and to 
formulate an optimal LSS, which is an alternative monitoring strategy.

\section{Methods}

\subsection{Patients and treatment regimens}

From March 2019 to April 2020, we collected 76 patients received allo-HSCT. This study was approved by the Beijing's Children Hospital and all patients/parents provided informed consent.

In the pre-treatment regimen, intravenous infusion of busulfan (BUSULFEX, Otsuka Pharmaceutical Co. LTD, Zhejiang, China) typically started eight days prior to transplantation. The infusion frequency was once every six hours within 3-4 days and each infusion process last for two hours. The dosage of Bu was based on weight $(1 \mathrm{mg} / \mathrm{kg}$ for children less than $9 \mathrm{~kg}, 1.2 \mathrm{mg} / \mathrm{kg}$ for children within a $9-16 \mathrm{~kg}$ range, $1.1 \mathrm{mg} / \mathrm{kg}$ for children between $16-23 \mathrm{~kg}, 0.95 \mathrm{mg} / \mathrm{kg}$ for children between $23-34 \mathrm{~kg}$, and $0.8 \mathrm{mg} / \mathrm{kg}$ for children more than $34 \mathrm{~kg}$ ) in accordance with the European Medicines Agency (EMA) (26). Different conditioning regimens were used dependent on the primary diseases and the types of donor. Briefly, Bu combined with cyclophosphamide (CTX) were the basic components of conditioning chemotherapy. For patients with malignant diseases, regimens containing cytarabine (Ara-C) were commonly applied, while fludarabine (FLU) was administrated for myeloablative treatment of patients with non-malignant tumors. Specific dosage and duration of different conditioning regimens have been exhibited in Supporting Information Table 1. Phenytoin (PHT) started 30 minutes before the initiation of Bu therapy in order to prevent central nervous system toxicity caused by Bu. Moreover, cyclosporine (CsA) with or without methotrexate (MTX) / mycophenolate mofetil (MMF) was given as GVHD prophylaxis.

\section{$2.2 \mathrm{Bu}$ determination and genotyping}

Blood samples for PK analysis and genotyping were withdrawn from central venous lines, in heparinized glass tubes, pre-infusion, $0.5,1,2,2.5,4$ and 6 hours after the first infusion. Plasma concentrations of Bu were determined using a high performance liquid chromatography-tandem mass spectrometry (HPLC-MS/MS) (1). The lower limit of quantitation was $10 \mathrm{ng} / \mathrm{mL}$ and the range of quantitation was from 10 to 10000 $\mathrm{ng} / \mathrm{ml}$. Pre-transplant genomic DNA was isolated and extracted from whole blood or hemocyte prior to the first Bu infusion. GST genotypes of patients, GSTA1 (rs3957356 and rs3957357, which defines haplotype ${ }^{*} A$ and ${ }^{*} B$ ) and GSTM1 (rs3754446), were detected with the ABI 3730XL DNA Analyzer (ABICo.; BioSune Biotechnology Co., Ltd, Shanghai, China). Supporting Information Table 2 displays the primer sets and Tm used for the genotyping assays.

\subsection{PPK analysis}

BU plasma concentration-time data was analyzed using a Non-Linear Mixed-Effects (NLME) model implemented in Phoenix 8.0 (Certara USA Inc., Princeton, NJ, USA). Typical PPK parameters and their random inter-individual variability (IIV) were estimated using a First-order Conditional Estimation method with Extended Least Squares method (FOCE ELS). One- or two-compartment distribution with first-order elimination were tested as structural model. Exponential errors follows a log-normal distribution, assumed to describe the IIV in PK parameters by the equation $P_{i}=P \times e^{\eta i}$, where $P_{i}$ is the individual PK parameter of the $i$ th individual, $P$ is the geometric average population value, and $\eta_{l}$ is the subject-specific random effect value, normally distributed random variable with a mean of 0 and a variance of $\omega^{2}(27)$. Additive, proportional, combined additive and proportional models were evaluated to account for the intra-individual (residual) variability. The selection of base model was based on changes in -2 log likelihood (-2LL) and on graphic analyses of the Goodness-of-fit (GOF).

Based on scatter-plots of individual parameters versus covariates and clinical plausibility, we selected candidate covariates for each PK parameters (28). The influence of potential covariates [sex, age, body weight, body surface area (BSA), alanine transaminase (ALT), aspartate aminotransferase (AST), alkaline phosphatase (ALP), total bilirubin (TBIL), creatinine (CRE), creatinine clearance (CLcr), GSTA1 genotypes $\left({ }^{*} A /{ }^{*} A,{ }^{*} A /{ }^{*} B,{ }^{*} B /{ }^{*} B\right)$, GSTM5 genotypes, primary disease (malignant disease, non-malignant disease) and drug interactions (FLU)] on clearance (CL) and volume (V) were further investigated using a stepwise 
procedure. Categorical covariates were coded as number, and continuous covariates were centered on their median value (27). During forward selection, covariates were defined as significance if the -2LL decreased by at least 3.84 ( $p[?]$ 0.05) following their inclusion in the model. During backward elimination, one covariate could remain in the final model if the -2LL increased by at least 6.63 ( $p$ [?] 0.01$)$ when removed at a time from the full model.

\subsection{Model validation}

Shrinkage in individual random effects were evaluated in order to assess whether the final model could be capable to estimate individual PK parameters by taking advantage of population typical values and sparse PK data. Shrinkage values of less than $20 \%$ indicate that the individual data are rich enough to compute the PK parameters, whereas larger shrinkage values generally mean that individual Bayesian estimates are biased towards the population mean values(29).

Graphical observation of the final model adopted GOF, including conditional weighted residuals (CWRES) over population predicted concentrations (PRED) or time after dose (TAD) and the relationship between observed (OBS) and PRED or individual predicted value (IPRED) (30). In addition, the final model was evaluated using visual predictive check (VPC) and bootstrap analysis. VPC was based on the final pharmacokinetic estimates and then calculated the $95 \%$ confidence interval (CI) for concentrations by simulating 1,000 individuals. Simulated concentrations were compared with the 5 th, 50 th, 95 th percentiles of the observed concentrations. In the bootstrap analysis (31), the $95 \%$ CI of the parameter estimates were derived from 1000 datasets of 69 subjects generated by random sampling using the Phoenix NLME.

External validation of the model was performed using an external dataset to evaluate the predictive performance of the final PPK model. The external dataset consisted of 81 busulfan concentrations from 14 children undergoing allo-HSCT. Busulfan plasma concentrations were predicted by fixing the population PK parameters to the final estimates of the previously established model and setting maximum evaluations to 0 . From this study, measured concentrations of individual patients assigned to busulfan were compared with calculated concentrations of these individual patients at the same time with our PPK model using their BSA, ALT and GSTA1 genotypes of patients. Differences of $<20 \%$ between calculated and measured concentrations were allowed (32).

\subsection{Dosing Simulations}

A new dosage scheme using a simulated dataset of 1000 patients was designed to achieve a targeted AUC of $1125 \mu \mathrm{M} \cdot \mathrm{min}$. The BSA of simulated patients were distributed from $0.2 \mathrm{~m}^{2}$ to $1.6 \mathrm{~m}^{2}$. Meanwhile, the genotypes of GSTA1 included ${ }^{*} A{ }^{*} A$ and ${ }^{*} A * B$ and the range of AST was coincident with the clinical dataset. Then, $1000 \mathrm{AUC}_{\text {simulated }}$ values and their variabilities were generated in Phoenix-NLME. Model-based doses were recommended according to the following Equation 1 :

$$
\overline{\text { Dose }_{\text {recommended }}=1125 / \mathrm{AUC}_{\text {simulated }} \times \text { Dose }_{\text {simulated }} \quad(1)}
$$

Then, AUC values were calculated according to the new recommended doses. Variabilities of AUC were shown by the range between minimum and maximum values and the coefficient of variation $(\mathrm{CV})$. The rate of achieving the targeted AUC window (900-1350 $\mu \mathrm{M} \cdot \min )$ was also listed.

\subsection{Optimized Sampling Scheme}

The final PPK model provides information on typical PK parameters and variabilities associated with these values as well as how specific covariates (e.g. BSA, AST, genotypes) influence the PK of BU. After evaluating final model predictive performance, filtrating optimal LSS was considered to optimize the sampling scheme for TDM. Candidate sampling points included: (1) 2, 2.25, 4 and 6 hours; (2) 2, 2.5, 4 and 6 hours; (3) 2.25, 2.5, 4 and 6 hours; (4) 2, 4 and 6 hours; (5) 2.25, 4 and 6 hours; (6) 2.5, 4 and 6 hours; (7) 2, 2.25 and 4 hours; (8) 2, 2.25 and 6 hours; (9) 2, 2.5 and 4 hours; (10) 2, 2.5 and 6 hours; (11) 2.25, 2.5 and 4 hours; (12) 2.25, 2.5 and 6 hours; (13) 2 and 4 hours; (14) 2 and 6 hours; (15) 2.25 and 4 hours; (16) 2.25 and 
6 hours; (17) 2.5 and 4 hours; (18) 2.5 and 6 hours after the beginning of first infusion. The blood drug concentration at 2.25 hours after administration was simulated by the final model. Each candidate LSS was tested by Bayesian method to obtain $\mathrm{AUC}_{0-6 \mathrm{~h}}$ of each patient. $\mathrm{APE}, \mathrm{MAPE}$ and $\mathrm{RMMSE}$ were calculated by comparing the predicted $\mathrm{AUC}_{0-6 \mathrm{~h}}$ derived from the LSS with the actual $\mathrm{AUC}_{0-6 \mathrm{~h}}$ obtained from each patient's full concentration-time samples, as according toEquation 2, 3 and 4 to evaluate predictive accuracy:

\begin{tabular}{|c|c|c|}
\hline $\operatorname{APE}(\%)=\underline{\mid \text { predicte }}$ & $\frac{d \mathrm{AUC}_{0-6 h}-\text { actual } \mathrm{AUC}_{0-6 h} \mid}{\text { actual } \mathrm{AUC}_{0-6 h}} \times 100 \%$ & $(2)$ \\
\hline $\operatorname{MAPE}(\%)=\frac{1}{N}$ & $\left(\frac{\mid \text { predicted } \mathrm{AUC}_{0-6 h}-\text { actual } \mathrm{AUC}_{0-6 h} \mid}{\text { actual } \mathrm{AUC}_{0-6 h}} \times 100 \%\right)$ & $(3)$ \\
\hline $\operatorname{rRMSE}(\%)=$ & $\left(\frac{\text { predicted } \mathrm{AUC}_{0-6 h}-\text { actual } \mathrm{AUC}_{0-6 h}}{\text { actual } \mathrm{AUC}_{0-6 h}} \times 100 \%\right)^{2}$ & $(4)$ \\
\hline
\end{tabular}

The lower the MAPE value, the better the LSS. A sampling strategy was considered to display a good predictive performance when the $95 \%$ confidence interval around the MAPE was less than $20 \%$ of the reference $\mathrm{Bu} \mathrm{AUC}_{0-6 \mathrm{~h}}$ values (33). Bland-Altman plots were applied to assess the agreement between the predicted and the actual $\mathrm{AUC}_{0-6 \mathrm{~h}}$, and the difference was expressed as a mean $\pm 1.96 \mathrm{SD}$.

\subsection{Definition of Clinical Outcomes}

The definition of engraftment was absolute neutrophil count (ANC) [?] $0.5 \times 10^{9}$ per liter for the first three days in a row and platelet count (PLT) [?] $20 \times 10^{9}$ per liter for the first one week after HSCT $(10,16)$. Primary engraftment failure was defined as failing to reach an ANC of $0.5 \times 10^{9} / \mathrm{L}$ within 30 days after HSCT (16). In brief, the diagnosis of SOS was according to the modified Seattle criteria (34). The Mount Sinai Acute GVHD International Consortium (MAGIC) was taken as the diagnostic criterion for aGVHD (35).

\subsection{Statistical Analysis}

Continuous data, like pharmacokinetics parameters, was compared using a two-tailed t-test, whereas timeto-event data was compared by the log-rank test. Univariate analysis was performed with GraphPad Prism (GraphPad Software, San Diego, CA). In this study, $p$ values $<0.05$ are considered as statistical significance, and $p$ values $<0.1$ were deemed as trends.

\section{Results}

\subsection{Population characteristics}

From March 2019 to April 2020, 76 patients underwent allogeneic HSCT and received IV busulfan. The GSTA1 genotypes for four patients and GSTM1 genotypes for six patients were missing because of samples being unavailable or detection failure. GSTA1-52C and $T$ defined haplotype ${ }^{*} A$ and ${ }^{*} B$, respectively. In relation to $G S T A 1-52 T / C$ and $-69 A / G$ SNPs, a higher percentage of $C C / G G(78.08 \%)$ versus $T T / A A$ $(1.37 \%)$ and $C T / G A(20.55 \%)$ was found in our model-building patient cohort. There was only one patient with GSTA1 ${ }^{*} B^{*} B$. Thus, 69 patients were included in our final PPK analysis. Table 1 summarizes characteristics of patients including demographics, primary diseases, donor types and conditioning regimens. Supporting Information Table 3 further elaborates patients' diagnoses. The genotyping results are shown inTable 2 .

\subsection{PPK model}

A total of 398 blood samples were collected for the PPK analysis.Supporting Information Figure 1 plotted concentration-time profiles of Bu. A one-compartment model with first-order elimination was suitable to describe the profiles of busulfan pharmacokinetics, and the exponential model was feasible to estimate the inter-individual variability. Neither proportional error model nor additive error model could perform well, while a combined proportional and additive residual error model provided an adequate fit $(\Delta-2 \mathrm{LL}>20, p$ $<0.001$ ). 
In the forward model building step, BSA, AST, types of primary diseases and GSTA1 genotypes declined the value of -2LL by more than $3.84(p<0.05)$ at each addition. The GSTA1 mutation, BSA and the level of AST significantly affected the clearance (CL) of busulfan. Meanwhile, the volume (V) of busulfan was influenced by BSA and types of primary diseases, while the GSTM1 genotypes had no significant effect on PK parameters.

During backward elimination, the value of -2LL increased significantly $(\Delta$-2LL $>6.63, p<0.01)$ when respectively removing the GSTA1 mutation, BSA and the level of AST out of the model. Thus, the three covariates mentioned above could remain in the final model. Types of primary diseases were eliminated in the final model because it failed to significantly increase the $-2 \mathrm{LL}$ value $(\Delta-2 \mathrm{LL}=6.333, p>0.01)$. Table 3 presented the detailed PK parameters of the base and final model. The CL and V of busulfan were affected by the GSTA1 genotypes, BSA and AST as illustrated by the following Equation $\mathbf{5}$ and $\mathbf{6}$ :

\begin{tabular}{|c|c|}
\hline 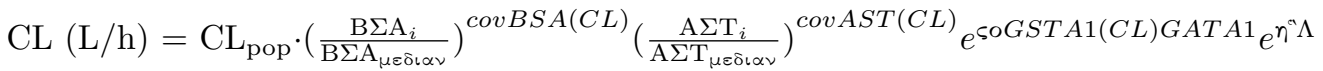 & (5) \\
\hline $\begin{array}{l}\mathrm{V}(\mathrm{L})=\mathrm{V}_{\text {pop }} \\
\text { selectlanguagegreek }\left(\mathrm{BSA}_{i} \frac{\left.\overline{\mathrm{BSA}}_{\text {median }}\right)^{\mathrm{covBSA}(V) e_{e}^{\mathrm{V}}}}{}\right.\end{array}$ & (6) \\
\hline
\end{tabular}

When patients carried the $G S T A 1{ }^{*} A /{ }^{*} B$ mutation, GSTA1 $=1$, and when patients carried the ${ }^{*} A /{ }^{*} A$ genotype, $G S T A 1=0$.

Population estimates for a patient with median BSA with $G S T A 1{ }^{*} A /{ }^{*} A$ were CL $4.79 \mathrm{~L} / \mathrm{h}$ and V $14.8 \mathrm{~L}$. The values of inter-individual variability for CL and V were $18.65 \%$ and $23.63 \%$, respectively. CL of patients carrying the $G S T A 1 * A /{ }^{*} B$ genotype was predicted to decline by $17.3 \%$ compared with those carrying GSTA1 ${ }^{*} A /{ }^{*} A$. As a result, CL/BSA between two genotypes had a significant difference $(p=0.0103)$, in spite of AUC/BSA without significant difference (Figure 1 ).

3.3 Model evaluation and validation

Shrinkage values of clearance and volume were respectively 0.207 and 0.130 , which manifested that the model-building dataset was rich enough to compute the PK parameters of busulfan in children.

Compared with the base model, the -2LL value of final model declined by 175.26 , which indicated that the model improved substantially after incorporating covariates, BSA, AST and GSTA1 phenotype. As demonstrated in the scatter plots of OBS vs. PRED (Figure 2A and 2B ), the PRED strongly deviated from the OBS in the base model, but PRED agreed with the OBS in the final model. In addition, the CWRES in the final model were more uniformly distributed within the accepted range $(y= \pm 2)$ than that in the base model (Figure 2C and 2D ). As a whole, the final model was visually improved in terms of the GOF and had a more accurate predictive performance.

The estimated values generated by bootstrap analysis were close to the parameters in the final model, which also fell within $95 \%$ CIs (Table 3 ). Thus, it can be considered that the final model was accurate and robust. Figure 3 clearly displayed that 5 th, 50th and 95 th percentiles of simulation values almost coincided with that of observed concentrations. The results of VPC combined with bootstrap analysis confirmed the exactitude of the parameter estimates and demonstrated the reliability of the final model. For external validation, a mean difference of $18.48 \%$ was observed in the simulated Bu concentrations generated using the established PPK model when compared with the observed Bu concentrations (Figure 4 ).

\subsection{New dosing strategy and simulations}

To achieve a target AUC of $1125 \mu \mathrm{M} \cdot \mathrm{min}$, a simple linear relationship between the total doses and BSA is illustrated in Figure 5(A). The linear Equation 7 and 8 for $G S T A 1{ }^{*} A /{ }^{*} A$ and ${ }^{*} A{ }^{*} B$ was respectively: 


\begin{tabular}{ll}
\hline Dose recommended $(\mathrm{mg})=34.14 \times \mathrm{BSA}\left(\mathrm{m}^{2}\right)+3.75$ & $(7)$ \\
\hline Dose $_{\text {recommended }}(\mathrm{mg})=30.99 \times \mathrm{BSA}\left(\mathrm{m}^{2}\right)+3.21$ & $(8)$ \\
\hline
\end{tabular}

The dosages were normalized by BSA $\left(\mathrm{mg} / \mathrm{m}^{2}\right)$ due to the fact that body size-based dosing is more familiar to pediatricians. As demonstrated in Figure 5 (B), the doses normalized by BSA were shown to be a log-linear function of BSA. Dosages according to this log-linear curve would be inconvenient for application in clinical, so a nomogram of BSA categories was derived. Three dosages for each genotype were then defined according to selected BSA categories in order for convenient clinical application. Finally, a new dosing strategy based on BSA normalization was listed in Table 4 .

BSA-based dosing recommended by the final PPK model was helpful for targeting the patient AUC. The rate of success in achieving the targeted AUC window (900-1350 $\mu \mathrm{M} \cdot \mathrm{min})$ was $99.58 \%$ in simulated patients. The new dosage yielded homogeneous AUC values in different BSA categories, and the CV of $7.57 \%$ in AUC was low.

\subsection{Limited sampling strategies}

Eighteen models with different sampling time points are all listed inTable $\mathbf{5}$. The relationship between the predicted and actual $\mathrm{AUC}_{0-6 \mathrm{~h}}$ for these models is shown in Supporting Information Figure 2 . From two to four-point models, $83.33 \%$ LSSs fitted well with the correlation $\left(r^{2}\right)$ of more than 0.85 . Prediction precision of LSSs expressed as rRMSE and MAPE is also given in Table $\mathbf{5}$. Model $2\left(\mathrm{C}_{2 \mathrm{~h}}, \mathrm{C}_{2.5 \mathrm{~h}}, \mathrm{C}_{4 \mathrm{~h}}\right.$ and

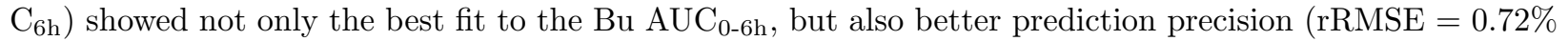
and MAPE $=4.55 \%)$ than other LSSs. Among the three-point models, Model $9\left(\mathrm{C}_{2 \mathrm{~h}}, \mathrm{C}_{2.5 \mathrm{~h}}\right.$ and $\left.\mathrm{C}_{4 \mathrm{~h}}\right)$ and Model $10\left(\mathrm{C}_{2 \mathrm{~h}}, \mathrm{C}_{2.5 \mathrm{~h}}\right.$ and $\left.\mathrm{C}_{6 \mathrm{~h}}\right)$ both behaved well, and with similar prediction precision to Model 2. Within these models, no patients had an $\mathrm{AUC}_{0-6 \mathrm{~h}}$ lower than $-15 \%$ or higher than $+15 \%$ of the reference value. As for these two-sampling schemes, Model $13\left(\mathrm{C}_{2 \mathrm{~h}}\right.$ and $\left.\mathrm{C}_{4 \mathrm{~h}}\right)$, Model $14\left(\mathrm{C}_{2 \mathrm{~h}}\right.$ and $\left.\mathrm{C}_{6 \mathrm{~h}}\right)$, Model $17\left(\mathrm{C}_{2.5 \mathrm{~h}}\right.$ and $\left.\mathrm{C}_{4 \mathrm{~h}}\right)$ and Model $18\left(\mathrm{C}_{2.5 \mathrm{~h}}\right.$ and $\left.\mathrm{C}_{6 \mathrm{~h}}\right)$ had relatively low rRMSE and MAPE. The Bland-Altman plots have verified the excellent capacity of prediction of the seven models above (Supporting Information Figure $3)$.

\subsection{Clinical outcomes}

Among the clinical outcomes analyzed, five patients died after HSCT, one of whom died of SOS and the other four died of severe infection. Graft failure occurred in two patients with $\mathrm{AUC}_{0-6 \mathrm{~h}}$ of 659.9 and $482.7 \mu \mathrm{M} \cdot \mathrm{min}$. Engraftment was achieved for $97.10 \%$ of patients (median time 12 days, range 10-19 days) for neutrophils within 30 days after transplantation and 59 patients achieved engraftment for platelets within a median of 15 days (range 7-30). As shown in Figure 6 , there were significant differences in ANC recovery and survival rate between patients with two GSTA1 genotypes $(p<0.05)$. Seven patients $(10.14 \%)$ developed SOS and aGVHD I-IV was documented in 57 patients (82.61\%), of whom seventeen had grade III-IV disease (24.64\%). The clinical outcomes of patients with two GSTA1 genotypes were compared in Supporting Information Table 4 .

In a univariate model, type of donor (HLA matched vs. mismatched) was the single variable that had significant association with the incidence of aGVHD $(p=0.022)$ (Figure 7$)$. The logistic regression model was again put into use to investigate the relationship between the incidence of aGVHD I-IV, aGVHD III/IV, $\mathrm{SOS}$ and $\mathrm{Bu} \mathrm{AUC}_{0-6 \mathrm{~h}}$, while Supporting Information Figure 4revealed that no correlation between $\mathrm{Bu}$ $\mathrm{AUC}_{0-6 \mathrm{~h}}$ and regimen-related toxicity or mortality was observed.

\section{Discussion}

This study developed the first PPK model for busulfan that successfully incorporated GSTA1 genotypes in Chinese pediatric population and partly explained the source of large variability of busulfan exposure, suggesting that GST genotyping would be necessary for optimization of pediatric Bu treatment. 
The literature until now has been controversial regarding the correlation between $\mathrm{Bu}$ clearance with genetic polymorphisms (36). There have been several researches supporting the positive association between GSTs polymorphisms and $\mathrm{Bu}$ clearance since 2016. In a pediatric multicenter study, Ansari et al. (37) reported that the activity of GSTA1 promoter was significantly descended in the case of ${ }^{*} B$ haplotype compared with*A haplotype, thus the correlation between GSTA1 genotypes and clearance was distinguished. In addition, GSTA1 diplotypes with slow metabolizing capacity were associated with higher incidence of SOS, aGVHD and combined treatment-related toxicity. Another study succeeded in incorporating the $G S T$ genetic variants (GSTA1) into a PPK model for Bu in a Caucasian pediatric population, and then tailored the dose according to the individual metabolic capacity (38). For Chinese adults, Yin et al. (16) concluded that patients with $G S T A 1{ }^{*} A /{ }^{*} B$ genotype possessed a higher $\mathrm{C}_{\max }$, higher AUC and lower clearance than the group with GSTA1 ${ }^{*} A /{ }^{*} A$ genotype. Furthermore, GSTA1 expression in young children has been reported to be higher than adults (19). In a recent study of Japanese pediatric population $(\mathrm{n}=20)$, Nishikawa et al. (39) stated the correlation between GST polymorphisms and clearance was distinguished.

In our two cohorts $(\mathrm{n}=84)$, the frequency of GSTA1 ${ }^{*} B$ haplotype (GSTA1-52T/-69A) was 11.9\% (MAF $=0.119$ ). In other words, a minority of patients had the ${ }^{*} B$ haplotype, of which $1.19 \%$ were homozygous $\left(G S T A 1{ }^{*} B /{ }^{*} B\right)$. Therefore, the MAF of Chinese patients was lower than that of global population (MAF $=0.306)$ taken from 1000 Genomes. Due to the significant discrepancy of the frequency of haplotype ${ }^{*} B$ between different populations, studies in Caucasians may not be able to represent that in the Asian population. Besides, in a study conducted in patients with acute myeloid leukemia, Yee et al. (40) found that $\mathrm{C}$ allele in the GSTM1 locus (rs3754446) was associated with decreased Bu AUC of first dose and lower disease-free survival. Thus, GSTA1 and GSTM1 genotypes were taken into consideration in the present study when exploring the influence of GST polymorphisms on Bu PK in Chinese children.

A population pharmacokinetic model of $\mathrm{Bu}$ was developed to test the influence of gene mutation on the pharmacokinetic characteristics. In the final model, the estimate value of $\mathrm{CL}$ was $4.79 \mathrm{~L} / \mathrm{h}$ and of $\mathrm{V}$ was $14.8 \mathrm{~L}$, consistent with those results in previous studies (38). The PPK model demonstrated that the GSTA1 polymorphisms were associated with $\mathrm{Bu}$ clearance. Patients carrying the $G S T A 1{ }^{*} A /{ }^{*} B$ genotype had a $17.3 \%$ lower clearance than those carrying $G S T A 1{ }^{*} A /^{*} A$, which is consistent with previous studies reporting that the presence of mutation allele probably resulted in the decreased activity of GST enzyme $(11,37)$. However, genetic variation in GSTM1 showed no significant impact on Bu CL, likely because the function of the GSTM1 enzyme involved in Bu metabolism was less than the GSTA1 enzyme.

As shown in Figure 1, there was a significant difference in clearance per body surface area between the two GSTA1 genotypes, but there was no significant difference in AUC per body surface area. These results were indeed confusing. As is well-known, AUC of drug administered intravenously is affected by the distribution, metabolism and excretion of drug, while CL is influenced only by metabolism and excretion. There are many complex physiological factors that affect drug distribution, especially in children. Thus, AUC may be not completely inversely proportional to CL. As shown in Supporting Information Figure 1, the distribution phase (0-2 hours after dosing) shown large heterogeneity between two genotypes, which may slightly interfere the statistical analysis of AUC. Nevertheless, in the eliminate phase, it was obvious that patients with GSTA1 ${ }^{*} A{ }^{*} A$ appeared faster clearance than those with $G S T A 1{ }^{*} A{ }^{*} B$. Generally, the non-obvious significance of $\mathrm{AUC}_{0-6 \mathrm{~h}}$ seemed reasonable. Moreover, the number of patients treated with busulfan with an AUC value of more than $900 \mu \mathrm{M} \cdot \mathrm{min}$ was eight. In other words, $88.4 \%$ of children with HSCT could not reach the range of 900-1350 $\mu \mathrm{M}$-min recommended by FDA. The main factor resulting in the low $\mathrm{AUC}_{0-6 \mathrm{~h}}$ may be the fact that, for Chinese patients, the frequency of $G S T A 1{ }^{*} A$ with high metabolizing capacity was much higher when compared to $G S T A 1{ }^{*} B$ with slow metabolizing capacity. Thus, the relatively low level of $\mathrm{AUC}_{0-6 \mathrm{~h}}$ in this study population was rational.

In the final PPK model constructed in this study, AST levels were negatively correlated with CL of Bu in pediatrics and CL declined $38.34 \%$ when AST increased from 12.7 to $127.4 \mathrm{U} / \mathrm{L}$. This is unsurprising because busulfan is mainly eliminated by the liver as previously described. This study divided the patients into two subpopulations (malignant and non-malignant diseases), which had no significant effect on the 
pharmacokinetic parameters of $\mathrm{Bu}$. In fact, malignant diseases could be subdivided into seven types according to pathology, and non-malignant diseases included six types. Nevertheless, some of the pathologies, such as osteopetrosis, were rare and present in only a small number of patients, resulting in an impossible comprehensive evaluation of variability of $\mathrm{Bu} \mathrm{PK}$. In oral busulfan-based pediatric research, the influence of underlying diseases on busulfan disposition was significant and CL/F was significantly lower in group with immune deficiencies than other groups (metabolic diseases, hemoglobinopathies and hematological malignancies) (25). Hence, the number of different types of cases needs to be expanded in order to analyze the specific impact of every primary disease on Bu PK.

There are theoretical and documented medication interaction with fludarabine and busulfan (41). Clearance of IV $\mathrm{Bu}$ decreased significantly in patients receiving concomitant fludarabine administration $(p=0.0016)$ and the average of reduction was 9.7\% (42). However, there was no other studies drawing similar conclusions $(43,44)$. Not surprisingly, in this PPK model, the inclusion of fludarabine as a covariate failed to significantly decline the value of -2LL during the forward selection. In fact, patients in our cohort received one of the two regimens, $\mathrm{Bu} / \mathrm{CTX} / \mathrm{FLU}$ and $\mathrm{Bu} / \mathrm{CTX} /$ Ara-C, as basic conditioning therapies. Due to the use of Bu/CTX in all patients without exception, the influence of CTX has already existed in the model. Although we only regarded FLU as a candidate covariate, in fact the influence of Ara-C has been also reflected in the model. When FLU=1, the impact of FLU was added to the model, and when FLU=0, the effect of Ara-C was included.

BSA was the most predictive covariate for CL and V, explaining $25.50 \%$ and $24.17 \%$ of the observed IIV, respectively. Weight-based dosing schedules were calculated with five fixed doses, however the model established by this study showed that body weight is not a significant predictor of $\mathrm{Bu} \mathrm{PK}$ in children. Additionally, in a retrospective study, SOS and early infectious complications occurred more frequently in the weight-based dosing group (45). Besides, a PPK model, developed among patients of all ages, revealed that the maturation of Bu clearance reaches half of adult values at 6 weeks after birth (46). Also, in children, Bu concentration did not show an obvious trend of change with postnatal age, which strongly supported the conclusion drawn from our cohort that age was not a significant factor affecting Bu clearance. According to the PPK model established with Chinese pediatric patients, BSA-based dosing scheme was recommended. When compared with weight-based dosage, the new dosing scheme not only took the influence of GSTA1 polymorphism into consideration, but could also be applied to patients with different liver function. More importantly, the simulation analysis demonstrated that the three fixed doses given on an $\mathrm{mg} / \mathrm{m}^{2}$ basis enabled almost all of the young patients $\left(0.2^{\sim} 1.6 \mathrm{~m}^{2}\right)$ to achieve the target $\mathrm{AUC}_{0-6 \mathrm{~h}}$. Since this new dosing regimen was based on a retrospective analysis, a prospective study is necessary to confirm the benefits in terms of efficacy and safety.

Based on the final PPK model, Bu Bayesian estimation of individual $\mathrm{AUC}_{0-6 \mathrm{~h}}$ values were performed by using various combinations of 2-4 sampling times within 6 hours following busulfan administration. Until this point, several LSS strategies have been established to predict BU exposure. Most LSS strategies published were estimated by using the trapezoidal rule (TR) or multiple linear regression (MLR), which usually reduced the accuracy of estimation and lacked professionalism. For example, Vaughan et al. (47) concluded that 4-5 sampling points $(3,4,5$ and 6 hours or $2,3,4,5$ and 6 hours after dosing) could predict well in adult patients receiving IV Bu four times daily. Teitelbaum et al. (48) developed an LSS with four sampling points (0, 2, 3 and 4 hours after the start of the second infusion). To achieve a more accurate estimate of AUC of every LSS, PPK models, considered to be the gold standard, should be applied. In this study population, $\mathrm{C}_{\max }$ generally appeared in 2-2.5 hours after dosing according to the concentration-time curve. Therefore, peak concentration was picked among $\mathrm{C}_{2 \mathrm{~h}}, \mathrm{C}_{2.25 \mathrm{~h}}$ or $\mathrm{C}_{2.5 \mathrm{~h}}$, and $\mathrm{C}_{4 \mathrm{~h}}$ and $\mathrm{C}_{6 \mathrm{~h}}$ were regarded as time points of elimination phase. Since the metabolism of busulfan conformed to the one-compartment model, selecting $\mathrm{C}_{4 \mathrm{~h}}$ or $\mathrm{C}_{6 \mathrm{~h}}$ on behalf of elimination phase had a similar predictive function. Model $9\left(\mathrm{C}_{2 \mathrm{~h}}, \mathrm{C}_{2.5 \mathrm{~h}}\right.$ and $\left.\mathrm{C}_{4 \mathrm{~h}}\right)$ and Model $10\left(\mathrm{C}_{2 \mathrm{~h}}\right.$, $\mathrm{C}_{2.5 \mathrm{~h}}$ and $\mathrm{C}_{6 \mathrm{~h}}$ ) elevated the accuracy and precision of prediction while increasing medical costs and pain for children. Model $13\left(\mathrm{C}_{2 \mathrm{~h}}\right.$ and $\left.\mathrm{C}_{4 \mathrm{~h}}\right)$ not only had a better predictive performance by rRMSE, MAPE and Bland-Altman analysis, but was also more in line with the clinical requirement of reducing sampling points for TDM. Finally, considering the accuracy of prediction and the feasibility of pediatric clinical practice 
synthetically, Model 13 was selected as the optimal LSS.

The relationship between pharmacokinetics and outcome or toxicity of busulfan reported previously are summarized in Supporting Information Table 5 . A multicenter retrospective research stated that graftfailure occurred more frequently in the low AUC group, meanwhile, acute toxicity and TRM were significantly higher in the high AUC group (5). Additionally, Andersson et al. (49) found that the probability of developing aGVHD increased with increasing AUC. Similar correlations between toxicities and AUC have been verified in both Korean children and adult groups $(14,50)$. However, no relationship between busulfan PK and toxicity was observed. Based on the data of 27 children with sickle cell disease undergoing HSCT, hepatic toxicity (SOS) was not associated with busulfan AUC (51). Moreover, Jessicaet al . (52) concluded that there was no significant association between AUC dose1 and death, relapse, or a composite of the two.

In terms of ANC recovery and survival rate, there were significant differences between patients with two GSTA1 genotypes $(p<0.05)$ in our study. Till now, no research has been reported concerning the relationship between GSTs polymorphisms and engraftment or mortality. However, Ansari et al. reported that GSTA1 ${ }^{*}$ B haploid was associated with higher incidence of treatment-related toxicity (37). Hence, we deduced that the high incidence of toxicity was likely connected to the postponement and the low rate of ANC recovery, which further led to higher chance of infection, the main cause of death in this study. In the next stage, we will include more abundant samples to verify the above conjecture.

In this study population, type of donor was the major predictor of the occurrence of aGVHD, whereas neither GSTM1 genotypes nor pharmacokinetic parameters were found to be significantly correlative to SOS. These results are difficult to interpret because multiple pre-transplantation and transplantation-related factors have been implicated in the rate of engraftment and the incidence of toxic effect. So far, no single factor has been reported to result in aGVHD or SOS independently. On one hand, it is worth noting that the homogeneity of patients' disease would be beneficial to the correlation analysis. For example, Srivastava at al. (53) concluded that GSTM1-null genotype was relevant to the incidence of SOS in 114 patients undergoing HSCT with uniform disease, $\beta$-thalassemia. On the other hand, the influence of complex combined medicines could not be ignored. Sixty-eight out of 69 patients adopted a combination of three or more myeloablative drugs and $57.97 \%$ of pediatric patients used four drugs with myeloablative activity in succession, which led that the AUC of a single drug, Bu, may be not the only influencing factor in curative effect or toxic reaction. It should be also recognized that when $\mathrm{Bu}$ was combined with different agents, relationships between $\mathrm{Bu}$ PK and outcome or toxicity would be varied (54), as has been confirmed in BU/CY/TBI regimens (55). Consequently, in order to clarify the correlation, analysis should be conducted according to disease types and conditioning regimens in the further.

\section{CONCLUSION}

In summary, this study was the first PPK model for busulfan that successfully incorporated GSTA1 genotypes in a Chinese pediatric population and partly explained the source of large variability of busulfan exposure, suggesting that TDM would be necessary for optimization of pediatric Bu treatment. Moreover, a BSA-based dosing regimen is recommended for individual busulfan therapy in order to weaken variability in busulfan exposure and to enhance the safety and efficacy of Bu treatment. Finally, an optimal LSS $\left(\mathrm{C}_{2 \mathrm{~h}}\right.$ and $\mathrm{C}_{4 \mathrm{~h}}$ ) would be convenient for TDM in the future.

\section{REFERENCES}

1. Jinjie Y, Sun N, Zhang S, Feng X, Chen X, Zhao D, et al. A rapid HPLC-MS/MS method for determining busulfan in hemolysis samples from children with hematopoietic stem cell transplantation. Biomedical chromatography : BMC. 2020:e4898.

2. ten Brink MH, Zwaveling J, Swen JJ, Bredius RG, Lankester AC, Guchelaar HJ. Personalized busulfan and treosulfan conditioning for pediatric stem cell transplantation: the role of pharmacogenetics and pharmacokinetics. Drug Discov Today. 2014;19(10):1572-86.

3. Dix SP, Wingard JR, Mullins RE, Jerkunica I, Davidson TG, Gilmore CE, et al. Association of busulfan 
area under the curve with veno-occlusive disease following BMT. Bone Marrow Transplant. 1996;17(2):22530.

4. Geddes M, Kangarloo SB, Naveed F, Quinlan D, Chaudhry MA, Stewart D, et al. High busulfan exposure is associated with worse outcomes in a daily i.v. busulfan and fludarabine allogeneic transplant regimen. Biology of blood and marrow transplantation : journal of the American Society for Blood and Marrow Transplantation. 2008;14(2):220-8.

5. Bartelink IH LA, Elisabeth ML, et al . Association of busulfan exposure with survival and toxicity after haemopoietic cell transplantation in children and young adults: a multicentre, retrospective cohort analysis. Lancet Haematol . 2016;3(11):e526-e36.

6. Ayala E, Figueroa J, Perkins J, Kim J, Yue B, Riches M, et al. Myeloablative intravenous pharmacokinetically targeted busulfan plus fludarabine as conditioning for allogeneic hematopoietic cell transplantation in patients with non-Hodgkin lymphoma. Clinical lymphoma, myeloma \& leukemia. 2015;15(6):335-40.

7. Parmar S, Rondon G, de Lima M, Thall P, Bassett R, Anderlini P, et al. Dose intensification of busulfan in the preparative regimen is associated with improved survival: a phase I/II controlled, randomized study. Biology of blood and marrow transplantation : journal of the American Society for Blood and Marrow Transplantation. 2013;19(3):474-80.

8. Andersson BS, Valdez BC, de Lima M, Wang X, Thall PF, Worth LL, et al. Clofarabine \pm fludarabine with once daily i.v. busulfan as pretransplant conditioning therapy for advanced myeloid leukemia and MDS. Biology of blood and marrow transplantation : journal of the American Society for Blood and Marrow Transplantation. 2011;17(6):893-900.

9. Malar R, Sjoo F, Rentsch K, Hassan M, Gungor T. Therapeutic drug monitoring is essential for intravenous busulfan therapy in pediatric hematopoietic stem cell recipients. Pediatr Transplant. 2011;15(6):580-8.

10. Wall DA, Chan KW, Nieder ML, Hayashi RJ, Yeager AM, Kadota R, et al. Safety, efficacy, and pharmacokinetics of intravenous busulfan in children undergoing allogeneic hematopoietic stem cell transplantation. Pediatr Blood Cancer. 2010;54(2):291-8.

11. Czerwinski M, Gibbs JP, Slattery JT. Busulfan conjugation by glutathione S-transferases alpha, mu, and pi. Drug metabolism and disposition: the biological fate of chemicals. 1996;24(9):1015-9.

12. Gibbs JP, Czerwinski M, Slattery JT. Busulfan-glutathione conjugation catalyzed by human liver cytosolic glutathione S-transferases. Cancer research. 1996;56(16):3678-81.

13. Huezo-Diaz P, Uppugunduri CR, Tyagi AK, Krajinovic M, Ansari M. Pharmacogenetic aspects of drug metabolizing enzymes in busulfan based conditioning prior to allogenic hematopoietic stem cell transplantation in children. Current drug metabolism. 2014;15(3):251-64.

14. Choi B, Kim MG, Han N, Kim T, Ji E, Park S, et al. Population pharmacokinetics and pharmacodynamics of busulfan with GSTA1 polymorphisms in patients undergoing allogeneic hematopoietic stem cell transplantation. Pharmacogenomics. 2015;16(14):1585-94.

15. Abbasi N, Vadnais B, Knutson JA, Blough DK, Kelly EJ, O'Donnell PV, et al. Pharmacogenetics of intravenous and oral busulfan in hematopoietic cell transplant recipients. Journal of clinical pharmacology. 2011;51(10):1429-38.

16. Yin J, Xiao Y, Zheng H, Zhang YC. Once-daily i.v. BU-based conditioning regimen before allogeneic hematopoietic SCT: a study of influence of GST gene polymorphisms on BU pharmacokinetics and clinical outcomes in Chinese patients. Bone Marrow Transplant. 2015;50(5):696-705.

17. Hassan M, Ljungman P, Bolme P, Ringden O, Syruckova Z, Bekassy A, et al. Busulfan bioavailability. Blood. 1994;84(7):2144-50. 
18. Gibbs JP, Murray G, Risler L, Chien JY, Dev R, Slattery JT. Age-dependent tetrahydrothiophenium ion formation in young children and adults receiving high-dose busulfan. Cancer research. 1997;57(24):5509-16.

19. Gibbs JP, Liacouras CA, Baldassano RN, Slattery JT. Up-regulation of glutathione S-transferase activity in enterocytes of young children. Drug metabolism and disposition: the biological fate of chemicals. 1999;27(12):1466-9.

20. Ansari M, Rezgui MA, Theoret Y, Uppugunduri CR, Mezziani S, Vachon MF, et al. Glutathione Stransferase gene variations influence BU pharmacokinetics and outcome of hematopoietic SCT in pediatric patients. Bone Marrow Transplant. 2013;48(7):939-46.

21. Zwaveling J, Press RR, Bredius RG, van Derstraaten TR, den Hartigh J, Bartelink IH, et al. Glutathione S-transferase polymorphisms are not associated with population pharmacokinetic parameters of busulfan in pediatric patients. Ther Drug Monit. 2008;30(4):504-10.

22. Kusama M, Kubota T, Matsukura Y, Matsuno K, Ogawa S, Kanda Y, et al. Influence of glutathione S-transferase A1 polymorphism on the pharmacokinetics of busulfan. Clin Chim Acta. 2006;368(1-2):93-8.

23. Rocha V, Porcher R, Fernandes JF, Filion A, Bittencourt H, Silva W, Jr., et al. Association of drug metabolism gene polymorphisms with toxicities, graft-versus-host disease and survival after HLA-identical sibling hematopoietic stem cell transplantation for patients with leukemia. Leukemia. 2009;23(3):545-56.

24. Vassal G, Deroussent A, Challine D, Hartmann O, Koscielny S, Valteau-Couanet D, et al. Is 600 $\mathrm{mg} / \mathrm{m} 2$ the appropriate dosage of busulfan in children undergoing bone marrow transplantation? Blood. 1992;79(9):2475-9.

25. Bertholle-Bonnet V, Bleyzac N, Galambrun C, Mialou V, Bertrand Y, Souillet G, et al. Influence of underlying disease on busulfan disposition in pediatric bone marrow transplant recipients: a nonparametric population pharmacokinetic study. Ther Drug Monit. 2007;29(2):177-84.

26. Nguyen L, Fuller D, Lennon S, Leger F, Puozzo C. I.V. busulfan in pediatrics: a novel dosing to improve safety/efficacy for hematopoietic progenitor cell transplantation recipients. Bone Marrow Transplant. 2004;33(10):979-87.

27. Aouri M, Barcelo C, Guidi M, Rotger M, Cavassini M, Hizrel C, et al. Population Pharmacokinetics and Pharmacogenetics Analysis of Rilpivirine in HIV-1-Infected Individuals. Antimicrobial agents and chemotherapy. 2017;61(1).

28. Ette EI, Ludden TM. Population pharmacokinetic modeling: the importance of informative graphics. Pharmaceutical research. 1995;12(12):1845-55.

29. Karlsson MO, Savic RM. Diagnosing Model Diagnostics. Clinical Pharmacology \& Therapeutics. $2007 ; 82(1): 17-20$.

30. Hooker AC, Staatz CE, Karlsson MO. Conditional weighted residuals (CWRES): a model diagnostic for the FOCE method. Pharmaceutical research. 2007;24(12):2187-97.

31. Ette EI. Stability and performance of a population pharmacokinetic model. Journal of clinical pharmacology. 1997;37(6):486-95.

32. van der Galiën R, Boveneind-Vrubleuskaya NV, Peloquin C, Skrahina A, Touw DJ, Alffenaar JC. Pharmacokinetic Modeling, Simulation, and Development of a Limited Sampling Strategy of Cycloserine in Patients with Multidrug-/Extensively Drug-Resistant Tuberculosis. Clin Pharmacokinet. 2020;59(7):899-910.

33. Nankivell BJ, Gruenewald SM, Allen RD, Chapman JR. Predicting glomerular filtration rate after kidney transplantation. Transplantation. 1995;59(12):1683-9.

34. Jones RJ, Lee KS, Beschorner WE, Vogel VG, Grochow LB, Braine HG, et al. Venoocclusive disease of the liver following bone marrow transplantation. Transplantation. 1987;44(6):778-83. 
35. Harris AC, Young R, Devine S, Hogan WJ, Ayuk F, Bunworasate U, et al. International, Multicenter Standardization of Acute Graft-versus-Host Disease Clinical Data Collection: A Report from the Mount Sinai Acute GVHD International Consortium. Biology of blood and marrow transplantation : journal of the American Society for Blood and Marrow Transplantation. 2016;22(1):4-10.

36. Kim MG, Kwak A, Choi B, Ji E, Oh JM, Kim K. Effect of glutathione S-transferase genetic polymorphisms on busulfan pharmacokinetics and veno-occlusive disease in hematopoietic stem cell transplantation: A meta-analysis. Basic Clin Pharmacol Toxicol. 2019;124(6):691-703.

37. Ansari M, Curtis PH, Uppugunduri CRS, Rezgui MA, Nava T, Mlakar V, et al. GSTA1 diplotypes affect busulfan clearance and toxicity in children undergoing allogeneic hematopoietic stem cell transplantation: a multicenter study. Oncotarget. 2017;8(53):90852-67.

38. Nava T, Kassir N, Rezgui MA, Uppugunduri CRS, Huezo-Diaz Curtis P, Duval M, et al. Incorporation of GSTA1 genetic variations into a population pharmacokinetic model for IV busulfan in paediatric hematopoietic stem cell transplantation. Br J Clin Pharmacol. 2018;84(7):1494-504.

39. Nishikawa T, Yamaguchi H, Ikawa K, Nakayama K, Higashi E, Miyahara E, et al. Influence of GST polymorphisms on busulfan pharmacokinetics in Japanese children. Pediatrics international : official journal of the Japan Pediatric Society. 2019;61(6):558-65.

40. Yee SW, Mefford JA, Singh N, Percival ME, Stecula A, Yang K, et al. Impact of polymorphisms in drug pathway genes on disease-free survival in adults with acute myeloid leukemia. Journal of human genetics. 2013;58(6):353-61.

41. Perkins JB, Kim J, Anasetti C, Fernandez HF, Perez LE, Ayala E, et al. Maximally tolerated busulfan systemic exposure in combination with fludarabine as conditioning before allogeneic hematopoietic cell transplantation. Biology of blood and marrow transplantation : journal of the American Society for Blood and Marrow Transplantation. 2012;18(7):1099-107.

42. Yeh RF, Pawlikowski MA, Blough DK, McDonald GB, O'Donnell PV, Rezvani A, et al. Accurate targeting of daily intravenous busulfan with 8-hour blood sampling in hospitalized adult hematopoietic cell transplant recipients. Biology of blood and marrow transplantation : journal of the American Society for Blood and Marrow Transplantation. 2012;18(2):265-72.

43. de Lima M, Couriel D, Thall PF, Wang X, Madden T, Jones R, et al. Once-daily intravenous busulfan and fludarabine: clinical and pharmacokinetic results of a myeloablative, reduced-toxicity conditioning regimen for allogeneic stem cell transplantation in AML and MDS. Blood. 2004;104(3):857-64.

44. Russell JA, Tran HT, Quinlan D, Chaudhry A, Duggan P, Brown C, et al. Once-daily intravenous busulfan given with fludarabine as conditioning for allogeneic stem cell transplantation: study of pharmacokinetics and early clinical outcomes. Biology of blood and marrow transplantation : journal of the American Society for Blood and Marrow Transplantation. 2002;8(9):468-76.

45. Gürlek Gökçebay D, Azik F, Ozbek N, Isik P, Avci Z, Tavil B, et al. Clinical comparison of weight- and age-based strategy of dose administration in children receiving intravenous busulfan for hematopoietic stem cell transplantation. Pediatr Transplant. 2015;19(3):307-15.

46. McCune JS, Bemer MJ, Barrett JS, Scott Baker K, Gamis AS, Holford NH. Busulfan in infant to adult hematopoietic cell transplant recipients: a population pharmacokinetic model for initial and Bayesian dose personalization. Clinical cancer research : an official journal of the American Association for Cancer Research. 2014;20(3):754-63.

47. Vaughan WP, Carey D, Perry S, Westfall AO, Salzman DE. A limited sampling strategy for pharmacokinetic directed therapy with intravenous busulfan. Biology of blood and marrow transplantation : journal of the American Society for Blood and Marrow Transplantation. 2002;8(11):619-24. 
48. Teitelbaum Z, Nassar L, Scherb I, Fink D, Ring G, Lurie Y, et al. Limited Sampling Strategies Supporting Individualized Dose Adjustment of Intravenous Busulfan in Children and Young Adults. Ther Drug Monit. 2020;42(3):427-34.

49. Andersson BS, Thall PF, Madden T, Couriel D, Wang X, Tran HT, et al. Busulfan systemic exposure relative to regimen-related toxicity and acute graft-versus-host disease: defining a therapeutic window for i.v. $\mathrm{BuCy} 2$ in chronic myelogenous leukemia. Biology of blood and marrow transplantation : journal of the American Society for Blood and Marrow Transplantation. 2002;8(9):477-85.

50. Lee JW, Kang HJ, Lee SH, Yu KS, Kim NH, Yuk YJ, et al. Highly variable pharmacokinetics of oncedaily intravenous busulfan when combined with fludarabine in pediatric patients: phase I clinical study for determination of optimal once-daily busulfan dose using pharmacokinetic modeling. Biology of blood and marrow transplantation : journal of the American Society for Blood and Marrow Transplantation. 2012;18(6):944-50.

51. McPherson ME, Hutcherson D, Olson E, Haight AE, Horan J, Chiang KY. Safety and efficacy of targeted busulfan therapy in children undergoing myeloablative matched sibling donor BMT for sickle cell disease. Bone Marrow Transplant. 2011;46(1):27-33.

52. Davis JM, Ivanova A, Chung Y, Shaw JR, Rao KV, Ptachcinski JR, et al. Evaluation of a Test Dose Strategy for Pharmacokinetically-Guided Busulfan Dosing for Hematopoietic Stem Cell Transplantation. Biology of blood and marrow transplantation : journal of the American Society for Blood and Marrow Transplantation. 2019;25(2):391-7.

53. Srivastava A, Poonkuzhali B, Shaji RV, George B, Mathews V, Chandy M, et al. Glutathione S-transferase M1 polymorphism: a risk factor for hepatic venoocclusive disease in bone marrow transplantation. Blood. 2004;104(5):1574-7.

54. Krivoy N, Hoffer E, Lurie Y, Bentur Y, Rowe JM. Busulfan use in hematopoietic stem cell transplantation: pharmacology, dose adjustment, safety and efficacy in adults and children. Current drug safety. 2008;3(1):606 .

55. Demirer T, Buckner CD, Appelbaum FR, Bensinger WI, Sanders J, Lambert K, et al. Busulfan, cyclophosphamide and fractionated total body irradiation for autologous or syngeneic marrow transplantation for acute and chronic myelogenous leukemia: phase I dose escalation of busulfan based on targeted plasma levels. Bone Marrow Transplant. 1996;17(4):491-5.

\section{Hosted file}

Table.docx available at https://authorea.com/users/359718/articles/481548-optimization-ofbusulfan-dosing-regimen-in-pediatric-patients-using-population-pharmacokinetic-modelincorporating-gst-polymorphisms

\section{Hosted file}

Figures.docx available at https://authorea.com/users/359718/articles/481548-optimizationof-busulfan-dosing-regimen-in-pediatric-patients-using-population-pharmacokinetic-modelincorporating-gst-polymorphisms 